\title{
Color Image Segmentation using Genetic Algorithm
}

\author{
Megha Sahu \\ M.Tech. Scholar \\ Department of Electronics and Communication \\ VNIT Nagpur, India
}

\author{
K.M. Bhurchandi \\ Professor \\ Department of Electronics and Communication \\ VNIT Nagpur, India
}

\begin{abstract}
This paper proposes color image segmentation approach and applying corresponding genetic algorithm under human vision limitations and capabilities. Most of the color image segmentation techniques initially use any clustering techniques to segment color images and then genetic algorithm (GA) is used only as optimization tool. Images are directly applied on 4D-color image histogram table using JND thresholds. The proposed algorithms are applied on Berkeley segmentation database in addition to general images. The segmentation performance of the proposed algorithms is estimated using Probabilistic Rand Index (PRI). The modified algorithm is proposed to improve the results and then compared with the proposed algorithm.
\end{abstract}

\section{Keywords}

RGB Color Model, JND threshold, 4D-histogram, Genetic algorithms, PRI

\section{INTRODUCTION}

Segmentation is considered as one of the most complex but important computer vision and image analysis tasks that has been extensively researched. Unavailability of a universal segmentation algorithm with performance matching to human vision especially for color image segmentation still keeps this field popular for research [1]. Image segmentation is always considered as the first step of analysis and understanding. Segmentation partitions an image into number of homogeneous non-overlapping regions. Color images have more information than grey-scale images and colors can be used to achieve better segmentation results. However it increases the complexity of the clustering problem due to increased color space dimensionality and also increased expectations due to enhanced human visibility. A way of handling the computational complexity is to use a directed search methods like genetic algorithms. Genetic algorithms $[2,3]$ mimic the process of evolution and have many qualities that make them suitable for image segmentation.

The partitioning approaches gradually lost their importance after the introduction of soft computing techniques. Most of the segmentation techniques provide approximate solution to the image segmentation problem, especially when applied to color image segmentation $[4,5,6]$. They further need optimization for enhancing the segmentation performance [7].

In this paper, we have presented a segmentation approach based on genetic algorithm and four dimensional Just Noticeable Difference (JND) color image histogram. Color corresponding to each bin in such obtained histogram is visually not similar from that of any other neighboring color bin as each bin represents only one shade of color [8] in the histogram. The modified approach to increase the segmentation results are used by implementation of HSI Color model. The JND color similarity measure is derived using human vision physiological capabilities and the Euclidean distance in the 3D RGB space [3].

Section 1 presents literature survey and introduction to the topic. Section 2 presents methods and algorithms including a brief human vision background, color models and 4D histogram along with a brief but lucid introduction to genetic algorithms. Section 3 presents the proposed algorithms and the related modifications made. Section 4 presents experimental results, comparison of modified results with the first approach, discussions and benchmarking with results of the other contemporary algorithms. Finally, section 5 presents conclusion.

\section{METHODS AND ALGORITHM 2.1. RGB Color Model}

Thomas Young presented his popular tricolor theory according to which all the human perceivable colors can be generated by appropriately adding the three basic colors Red $(\mathrm{R})$, Green (G), Blue (B) [8,9]. RGB color space is the default and most widely used color space. The RGB color model is an additive color model in which red, green, and blue basic lights are added together in various ways and combinations to reproduce a broad array of colors.

The name of the model comes from the initials of the three additive primary colors, red, green, and blue. The main advantage of RGB space is that it is the default color space for most of the image formats and hence the need for color space conversion is eliminated, saving computational time. The disadvantage is the perceptually non-uniformity i.e., the perceived difference between two colors and the Euclidean distance between two colors in the RGB space are not proportional [1]. The RGB color space is represented as in figure 1.

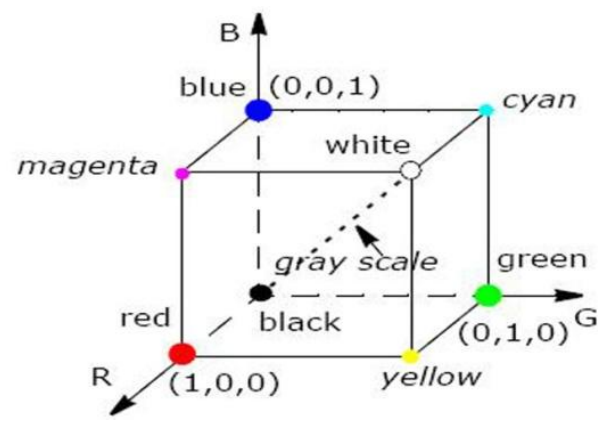

Fig 1: RGB Color Model

\subsection{HSI Color Model}

This color space is a deformation of RGB color cube. The three components of HSV are hue, saturation and value (also called intensity value). Hue is angle from nominal point around the circle to the color while saturation is the radius from central lightness or intensity axis to the color [1]. The main advantage of using HSI color model is, it represents 
colors similar to the human eye perception of colors [10]. The HSV color space is represented as in figure 2.

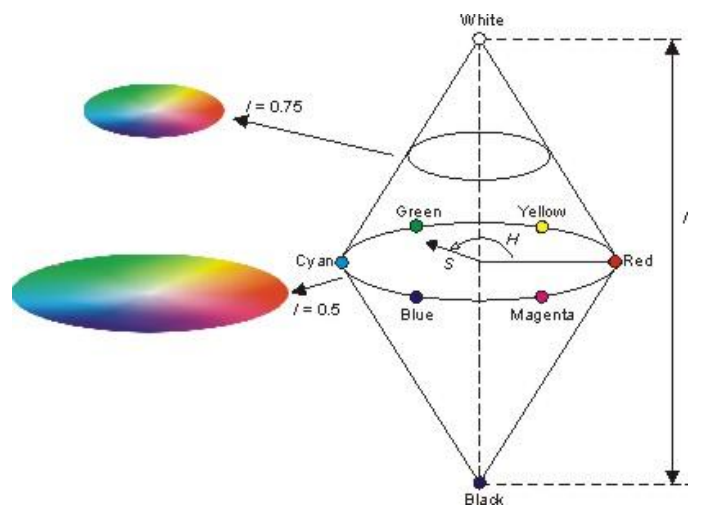

Fig 2: HSV Color Model

\subsection{JND Color Space}

JND is defined as the smallest difference between two colors which a person can detect. It is threshold below which any change in color cannot be detected by Human Visual System (HVS). In other words we can define that a human eye can distinguish between any two colors if the two colors are at least one JND away from each other. The human retina contains two basic types of light sensors namely; rods and cones, responsible for monochrome. There are three types of cones viz Red, Green and Blue. They respond to specific wavelength ranges corresponding to the three basic colors Red, Green and Blue. These cones provide a qualitative basis for representing a digital color image with RGB monochrome images. According to Thomas Young in three color theory, all other colors are perceived as linear combinations of these basic colors. The JND color space is obtained by sampling the RGB color space in nonlinear fashion [11]. According to Bhurchandi et al.[12] a vision observer (as defined by CIE) can respond to only 17000 colors at the maximum intensity without saturating the human eye. Generally, the human eye or brain requires a difference of at least three 'JND' [13] because it cannot perceive ' 1 JND' change in color. ' 3 JND' is also used as a color similarity threshold for color image segmentation.

The red cones present in the human retina are less sensitive, blue cones are moderately sensitive and the green cones are mostly sensitive. In this paper, to simulate the physiological fact, the red axis is quantized in 24 levels (as in Munsell Book) and the blue and green axes are quantized in 26 and 28 levels respectively to accommodate 17000 colors. A new color space $\mathrm{Jr}, \mathrm{Jg}$, Jb where $\mathrm{Jr}$, $\mathrm{Jg}$ and $\mathrm{Jb}$ are three nonuniformly sampled orthogonal axes which represent the JNDs on the respective $\mathrm{R}, \mathrm{G}$ and $\mathrm{B}$ axes are obtained. The non uniform sampling of color space considering the physiological limitations of human color vision results in reduction in number of shades of colors resulting in uniform JND color space. The reduction in number of colors leads to reduction of search space and hence improves convergence time considerably [12].Thus, the new space formed is perceptually uniform and has the advantages of uniform spaces in image analysis [5]. The color image segmentation technique in HSI color space by using JND is presented in [15].

\subsection{4-D Histogram}

In Digital Image Processing, the histogram is a 2-dimensional graph showing the number of pixels in an image at each different intensity value found in that image. For example consider figure 3.The histogram readily gives information about the global statistics of digital images. Histogram can be used for a number of image analysis and processing algorithms.

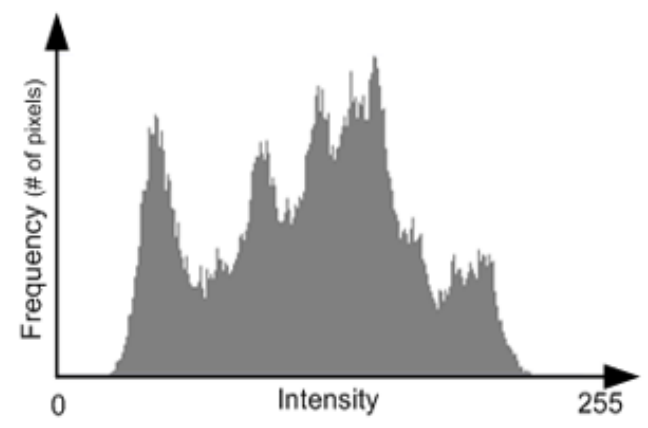

Fig 3: Grey Image Histogram

The algorithms and techniques proposed for gray images were initially applied to color images considering the RGB color images as combination of the three independent gray images in terms of $\mathrm{R}, \mathrm{G}$ and $\mathrm{B}$ intensities. Accordingly three independent histograms for three planes were plotted for $R, G$ and $\mathrm{B}$ colors. However such histograms do not carry any information about the frequency of any actual complex color triplet and also they can't make the relation between basic components of a complex color triplet. Thus many such algorithms failed due to the increased dimensionality of color space. The required color image histogram in fact is a four dimensional structure; three for the three basic colors R, G, B and fourth for the population of the complex color triplet. However, graphically plotting a four dimensional structure is almost impossible and even if somehow plotted it will be least useful for any analytical purpose. The importance of color image histogram is defined in $[11,12]$.

Thus, the 4D histogram is simply computed in the form of a table with four columns to accommodate R, G, B and population of the triplet. Thus the number of rows in the histogram table will be equal to number of different colors shades in a color image.

In this section, we present a definition of histogram of a color image in RGB space. The histogram of a given RGB image I $=f(1), f(2), f(3), f(4) \ldots \ldots, f(\mathrm{~m} \times \mathrm{n})$ is given by $\mathrm{H}(\mathrm{r}, \mathrm{g}, \mathrm{b})$ as in equation (1), where $m$ and $n$ are rows and columns of the image respectively and I represents the color intensity values. $\mathrm{N}$ is a frequency counter variable and $\mathrm{r}, \mathrm{g}, \mathrm{b}$ represents the color coefficients.

The histogram is a data structure or a table of size $\mathrm{Nx} 4$, where $\mathrm{N}$ indicates number of different colors present in the image. For practical purposes where the performance is expected to match that of human vision, the color image histograms needs to be sampled on $\mathrm{R} \mathrm{G}$ and $\mathrm{B}$ axis suitably to reduce the number of colors $[8,9]$.

Table 1. 4-D Color Image Histogram

\begin{tabular}{|c|c|c|c|}
\hline $\mathbf{R}$ & $\mathbf{G}$ & $\mathbf{B}$ & $\mathbf{H}(\mathbf{f})$ \\
\hline 79 & 25 & 123 & 206 \\
\hline 98 & 218 & 41 & 354 \\
\hline$\cdot$ & $\cdot$ & $\cdot$ & $\cdot$ \\
\hline $\mathrm{K}$ & 43 & 67 & 91 \\
\hline \multicolumn{3}{|c|}{ Total population } & mxn \\
\hline
\end{tabular}


The total population $(\mathrm{H})$ in Table-I must satisfy Equation (1).

$$
\sum H=m \times n
$$

The number of rows in Table-I is equal to the number of different complex color shades available in the image under the sampling conditions. In Table-1, there is one entry for each complex color. The color shades which are not present in the original image are not allotted any row in above Table-1. The color triplets are entered in the Table- 1 in the order of their presence in the image or as they are encountered during the scan of the image which starts scanning from the top leftcorner of the image. The total population $(\mathrm{H})$ in Table-I must satisfy equation (1).

\section{D-Histogram Algorithm}

1. We initialize the first row of the 4-D histogram table by entering the first top left corner pixel color triplet of the image [R G B] and the frequency (population) by one.

2. Then, we scan the image along the rows and read the next pixel color vector I $(m, n)$. We compute the frequency of each color pixel by the following procedure. For each pixel we compare it with its previous pixels to check if the Euclidean distance of the color triplet from any of the previous color triplets is less than or equal to the given threshold i.e., one JND (169), if it is less than the threshold we increment the population of that particular pixel color triplet by one, else we create a new row and add the RGB value of the color pixel and initialize its population by one.

3. We repeat the same procedure for each and every pixel encountered and finally, save the table for further applications of genetic algorithms.

So for this algorithm, any two colors with Euclidean distance less than or equal to 169 are considered the same color i.e.,

JND similar colors. This results in reduction in number of colors in a natural image and brings the number to a range suitable for image analysis in real time. As a result, handling of this image is easier in real time applications. Reconstruction of image based on the histogram obtained also yields a segmented image but the segmentation quality is poor. The visually different colors in Table 1 are further agglomerated using genetic algorithms discussed in the next section.

\subsection{Genetic Algorithm}

Genetic algorithm [16] is same as biological process evolution and have many qualities that make them suitable for optimization method. The process contains steps of division of fit chromosome population in two parts, natural selection of chromosomes and fitness of the chromosomes for further cross over to generate the offspring. A chromosome is made up of genes, each of that can represent a particular characteristic of an individual. Each individual in the population is given a fitness score that decides its suitability for further cross over. The higher the individuals fitness score, the greater is its probability of breeding. Cross over combines chromosomes of two individuals, creating a new individual which is different from either of the parents. The unfit chromosomes for cross over are expelled for further operation. The process is repeated till the expected optimal solution is reached. Since the more optimal individuals have a greater chance of breeding and participating in crossovers, the population leads to evolve and reach an optimal solution. However, GAs are not guaranteed to yield the best results. A general flow of genetic algorithms has been presented in figure 4 .

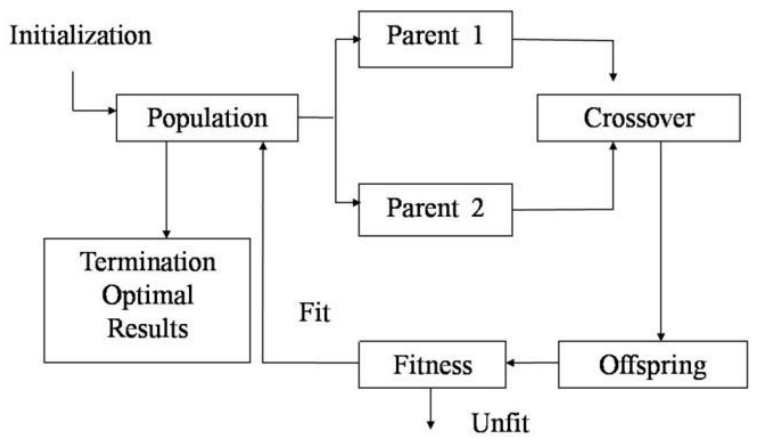

Fig 4: An evolutionary process like Genetic Algorithm

\section{PROPOSED ALGORITHMS}

The proposed algorithm is the approach used for color image segmentation where 4-D histogram is made and genetic algorithm is applied by dividing the histogram into two groups on the basis of population. The modified algorithm proposed after this approach yields better segmentation results on few of the images using HSI color space.

1. Initially classify the images into 4 categories and then apply the respective algorithms. The approach consists of Steps.

2. In this approach we used genetic algorithm with 4-D color image histogram technique.

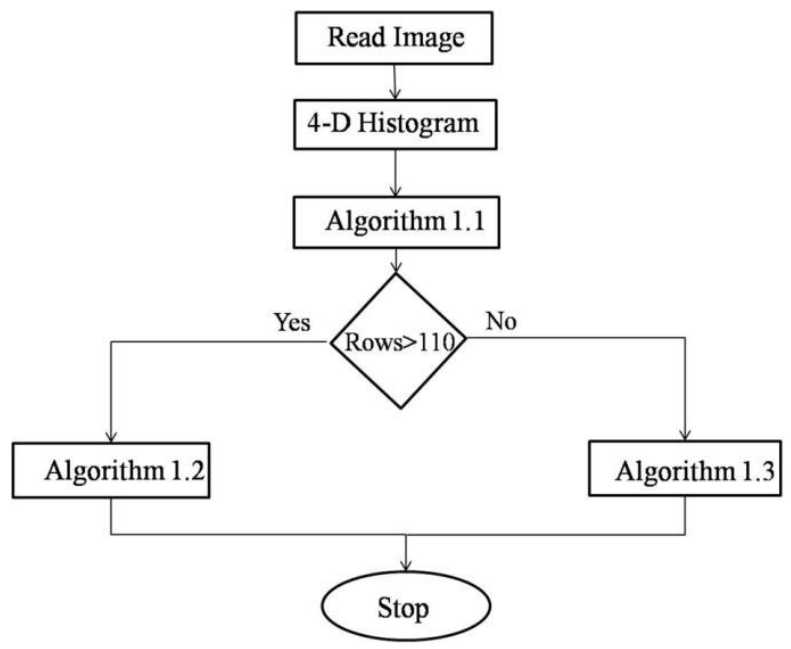

Fig 5: Flowchart of Proposed Approach

\section{Algorithm 1:}

Construction of 4-D color Histogram with JND threshold 169 using 4-D histogram algorithm.

\section{Algorithm 2:}

1. Arrange obtained histogram in descending order of population.

2. Calculate average value of population Hav.

$$
H_{a v}=\frac{m \times n}{u}
$$

3. Break obtained histogram into above average population (P1) and below average population (P2) based on average value obtained. 


$$
P_{1}=C \mid H_{C}>H_{a v} \text { and } P_{2}=C \mid H_{c}<H_{a v}
$$

4. Start crossover using 3 JND threshold.

Start from the first color in above average population P1 and check for each color in the below average population P2 for similarity (cross over) that is if a below average color is $\mathrm{w}$ to the first color then cross it with the first color of above average population by replacing with the color having highest population. Let $\mathrm{H} 1$ be population of color $\mathrm{C} 1$ in $\mathrm{P} 1$ and $\mathrm{H} 2$ be the population of the color $\mathrm{C} 2$ in $\mathrm{P} 2$; an offspring color is given as below; let $\mathrm{P} 1$ contains $\mathrm{i}$ colors and $\mathrm{P} 2$ contains $\mathrm{j}$ colors. Cr represents the color after the cross over function $\otimes$ for all $C_{1}(1: i) \in P_{1}$ and $C_{2}(1: j) \in P_{2}$

$$
\begin{aligned}
C_{r} & =C_{1} & & \text { for } H_{1}>H_{2} \\
& =C_{2} & & \text { for } H_{2}>H_{1}
\end{aligned}
$$

and

$H_{r}=H_{1} \otimes H_{2}=H_{1}+H_{2}$

At every crossover we eliminate the crossed over below average population color from the table.

5. Calculate percentage of population in first few rows of histogram

Here we initially classify an image according to its pixel population i.e., the percentage of population of pixel in image present in first specific number of rows of its histogram and then apply an appropriate genetic algorithm so that it yields better segmentation. The images are classified into two categories i.e., below $75 \%$ of total pixel population in an image in first specific number of rows and category 2 is between $75-100 \%$ total pixel population in image in first specific number of rows.

Algorithm 3: (Obtained population<75\%)

1. Similar to Algorithm 2 using 2.5 JND threshold for crossover.

2. Only change in algorithm:

1. Consider only the below average population group (P2) and calculate average population value.

2. Divide in two parts $\mathrm{P}_{21}$ and $\mathrm{P}_{22}$. Then doing crossovers on minimum distance criterion.

Let $\mathrm{i}$ and $\mathrm{j}$ be the pixel color groups such that $\mathrm{C} 1(1: \mathrm{i}) \in \mathrm{P} 21$ and $\mathrm{C} 2(1: \mathrm{j}) \in \mathrm{P} 22$. Let $\mathrm{n}$ represents a crossover iteration number variable.

for all $\mathrm{C} 1=1: \mathrm{i}$ and $\mathrm{C} 2=1: \mathrm{j}$

$C_{r}=C_{1} \quad$ for $H_{1}>H_{2}$

$=\mathrm{C}_{2} \quad$ for $\mathrm{H}_{2}>\mathrm{H}_{1}$

and

$H_{r}=H_{1} \otimes H_{2}=H_{1}+H_{2}$

Algorithm 4: (Obtained population > 75\%)

1. Repeating the algorithm 1 with different threshold of 3 JND and algorithm 4 with different (5.5 JND).

2. Only change:

1. Crossover on the basis of Energy also.

2. Convert RGB histogram to HSI histogram.

3. Take average value of saturation and intensity value.

4. Start crossover on histogram based only on hue with threshold 0.001 .
5. Again start crossover on histogram based only on hue with threshold 0.003 .

6. Convert back HSI to RGB.

7. Repeat the previous crossover steps till final histogram is obtained.

8. Calculate PRI.

Modification made in Algorithm 4: (Obtained population $>75 \%$ )

1. This approach is an improvement in the already proposed approach presented in previous chapter.

2. RGB histogram to HSI histogram.

3. Dividing HSV color model in 8 regions.

4. Taking average value of saturation and intensity values in these 8 regions getting different 8 thresholds in hue based on respective saturation and intensity values.

5. Again using these thresholds on obtained histogram.

6. At last doing crossover on obtained histogram based only on hue with threshold 0.003 .

7. Convert back to RGB.

8. Calculate PRI.

\section{EXPERIMENTAL RESULTS}

The proposed algorithms have been tested on the Berkley Segmentation Dataset (BSD)[17]. BSD contains 300 real life RGB images. It also contains benchmark segmentation result images manually obtained from 30 human subjects that is, multiple ground truth human segmentations of each image. In this section, we have presented visual results of some of the images out of 300 BSD images on the proposed variations of genetic algorithms presented in previous Algorithm and modified algorithm.

The results clearly depict that this algorithm works well for most of the real time images but it does not fit well for few images. The images from Fig 6(c), Fig 7 (c) and Fig 8(c) gives the better results than previous algorithm segmented images. The figures Fig 6(b), Fig 7(b) and Fig 8(b) are the outputs from previous approach. The average PRI for 300 images by using previous approach is calculated and it is found to be 0.789. And the average PRI (Probabilistic Rand Index)[18] for 300 images by modifying the previous approach is calculated and it is found to be 0.7924 . The average PRI of modified algorithm is good when compared to its previous algorithms shown in Table 2.

Comparison of results of previous approach 1 and modified approach 2 .

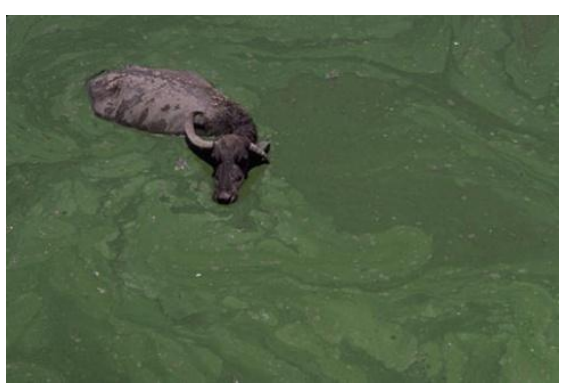

Fig 6(a): Input Image 


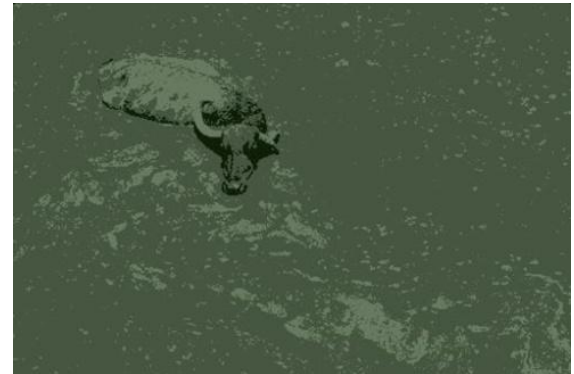

Fig 6(b): Output of Approach 1(PRI 0.811)

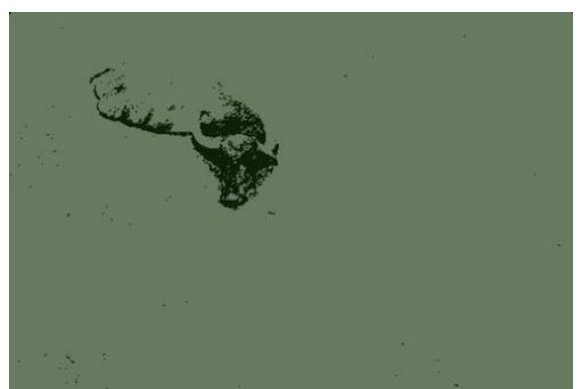

Fig 6(c): Output of Approach 2(PRI 0.9202)

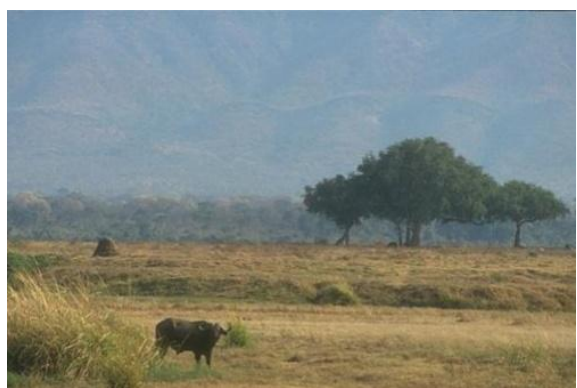

Fig 7(a): Input Image

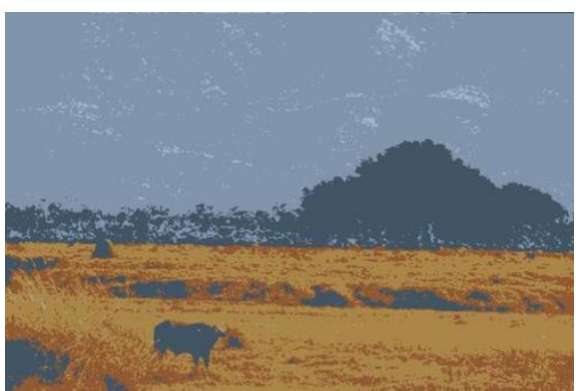

Fig 7(b): Output of Approach 1(PRI 0.8164)

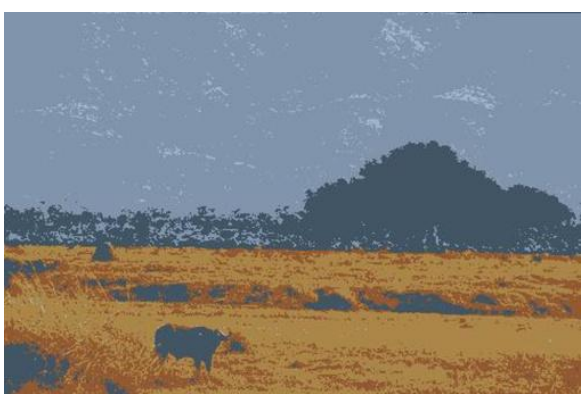

Fig 7(c): Output of Approach 2(PRI 0.8701)
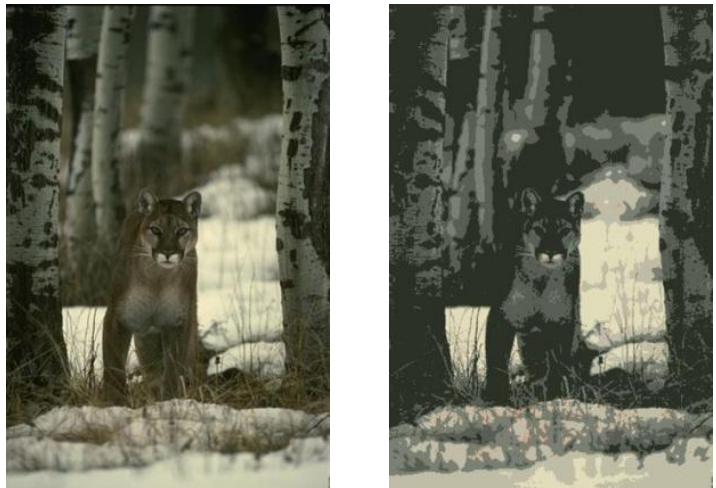

Fig 8(a): Input Image

Fig 8(b): Output of Approach 1

(PRI 0.5195)

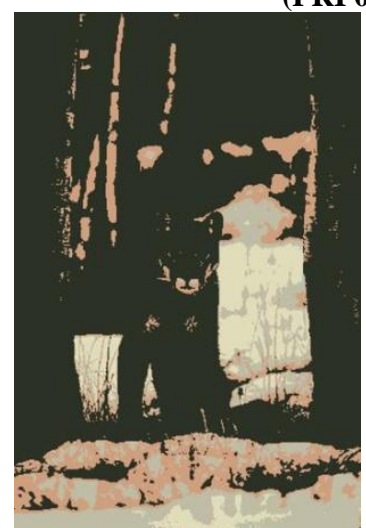

Fig 8(c): Output of Approach 2 (PRI 0.6986)

Table 2. Comparison of modified algorithm and Previous algorithm.

\begin{tabular}{|l|l|l|}
\hline Categories & PRI & PRI \\
\hline & $\begin{array}{l}\text { Previous } \\
\text { Algorithm }\end{array}$ & $\begin{array}{l}\text { Modified } \\
\text { Algorithm }\end{array}$ \\
\hline Category 1 & 0.80993 & 0.80993 \\
\hline Category 2 & 0.7896 & 0.78967 \\
\hline Category 3 & 0.78904 & 0.78904 \\
\hline Category 4 & 0.7725 & 0.7808 \\
\hline PRI of 300 images 0.7809 & $\mathbf{0 . 7 9 2 4}$ \\
\hline
\end{tabular}

Above Figures presents the results for three better segmented images. The GA using 4D-histogram faces few problems due to its randomness in crossovers, mutations and next generation crossovers. The maximum PRI obtained for an individual segmented images have been found to be as high as 0.98636 using the proposed family of algorithms using GAs for best optimization results.

\section{CONCLUSION}

From the above comparison we can say that the proposed approach is good for most of the real life available images. We also conclude by saying that color is very important feature of an object so we considered it as the only feature in the approach. The JND based sampling of RGB space has reduced the histogram computation time and decreased the color space dimensionality. HSI is better color space for color image segmentation. Of course, use of other features like, texture and shape with this algorithm may further improve the 
results. In future other features can also be included with pre and post processing methods for better segmentation outputs. Finally we conclude that 'A single algorithm is bound to fail while segmenting all types of real life images and scenes. Hence classification of images in some categories and then application of suitable algorithm to that particular category appears to be the only solution'.

\section{REFERENCES}

[1] M.Swain and D. Ballard, "Color indexing", International Journal of Computer Vision, Vol.7, no.1,pp.11-32,1991.

[2] B.Bhanu and S.Lee, "Adaptive image segmentation using a genetic algorithim", IEEE Transactions on Systems, Man and Cybernetics, vol. 25, no. 12, pp.15431567, December 1995.

[3] M. Farmer and D. Shrugas. "Application of genetic algorithims for wrapper-based image segmentation and classification", IEEE Congress on Evolutionary computation, pp.1300-1307, July 2006.

[4] M. Paulinas, Andrius Usinskas, "A Survey of genetic algorithm applications for image enhancement and segmentation", Information technology and control, ISSN 1392-12X, Vol.36,No.3,pp.278-284, 2007.

[5] Sang Ho Park, Il Dong Yun and Sang Uk Lee,"Color Image segmentation based on 3-D clustering: morphological approach", Elsevier, Pergamon, Pattern Recognition, Vol.44, No.8, pp. 1061-1076, 1998.

[6] S Praveena and IIa Vennila, " Optimization fusion approach for image segmentation using K-means algorithm", International Journal of Computer Applications, vol .2, no .7, pp.18-25, June 2010

[7] N Senthikumaran and R Rajesh, "Image segmentation- A survey of Soft computing approaches", Proceedings of International conference on Advances in Recent Technologies in Communiation and computing, pp.844846, 2009

[8] M. Farmer and D. Shrugas. "Application of genetic algorithms for wrapper-based image segmentation and classification", IEEE Congress on Evolutionary computation, pp. 1300-1307, July 2006.

[9] Petra Kudova, "Clustering Genetic Algorithm," IEEE, DOI10.1109/DEXA.2007.65, 2007.
[10] Kishor K. Bhoyar, "Performance enhancement of color based classification, and segmentation for image retrieval using JND approach", Ph. D. Thesis, Visvesvaraya National Institute of Technology, Nagpur, India, July 2010.

[11] R.B. Raut, K M Bhurchandi, "A Biologically inspired technique for sampling of color images", Proceedings of Bionetics 2008, Hyogo Japan, ACM digital Library, http://portal.acm.org/citation.cfm? id=1512523

[12] K M Bhurchandi, P M Nawghare, A K Ray, "An analytical approach For sampling the RGB color space considering physiological limitations of human vision and its application for color image analysis", Proceedings of ICGVIP2000, ACM digital Library., pp.44-49, 2000.

[13] N Senthikumaran and R Rajesh, "Image segmentation- A survey of Soft computing approaches", Proceedings of International conference on Advances in Recent Technologies in Communication and computing, pp.844846, 2009.

[14] A. Moghaddamzadeh and N. Bourbakis, "A fuzzy region growing approach for color images", Permagon, pattern recognition, Vol.30, No.6, pp.867-881, 1997.

[15] Gargi V. Sangamnerkar, Dr. K.K.Bhoyar "Color Image Segmentation in HSI Color Space Based on Color JND Histogram" International Journal of Image Processing and Visual Communication ISSN (Online) 2319-1724 : Vol.2, Issue 3, April 2014

[16] B. Bhanu and S. Lee, "Adaptive image segmentation using a genetic algorithm", IEEE Transactions on Systems, Man and Cybernetics, Vol. 25, No. 12 , pp.1543-1567, December 1995.

[17] Pablo Arbelaez, Charless Fowlkes, David Martin [DB/OL].http://www.eecs.berkeley.edu/Research/Project s/CS/vision/grouping/segbench/, 2007.

[18] R. Unnikrishnan, C. Pantofaru, and M. Hebert, "A measure for objective evaluation of image segmentation algorithms," in Proc. IEEE Int. Conf.Comput. Vision Pattern Recog. (CVPR), Workshop Empirical Evaluation Methods Comput.Vision, San Diego, CA, Vol. 3, pp. 3441, June 2005. 